\title{
Erratum to: Bowel perforations in a patient affected by Churg- Strauss syndrome under high-dose steroid treatment: will alternative drugs reduce risk of surgery?
}

Dario Venditti ${ }^{1} \cdot$ Valerio Balassone $^{1} \cdot$ Benedetto Ielpo $^{1}$. Oreste Buonomo $^{1} \cdot$ Giuseppe Petrella $^{1}$

Published online: 6 January 2016

(C) Springer-Verlag Berlin Heidelberg 2016

Erratum to: Rheumatol Int (2011) 31:1239-1241

DOI 10.1007/s00296-009-1289-5

The given and the surname of one of the co-authors' has been swapped in the original publication. The correct name should be Valerio Balassone.

The online version of the original article can be found under doi:10.1007/s00296-009-1289-5.

Benedetto Ielpo

benny078@libero.it

1 Department of Emergency Surgery, Policlinico Tor Vergata,

"Tor Vergata" University Hospital, Viale Oxford 81,

00133 Rome, Italy 